\title{
Butyric Acid Influences How $\beta$-Hydroxybutyrate Modulates Fatty Acid Oxidation and Ketogenesis To Support Ketosis
}

\author{
Emran Bashar ${ }^{1}$ and Franco Cavaleri ${ }^{1,2 *}$ \\ ${ }^{1}$ Biologic Pharmamedical Research, Canada \\ ${ }^{2}$ Experimental Medicine Program, University of British Columbia, Canada
}

Submission: August 28, 2018; Published: September 19, 2018

*Corresponding author: Franco Cavaleri, 688-2397 King George Blvd Surrey BC, V4A 7E9, Canada; Tel: +1-855-518-8858;

Email: franco.c@biologic-med.com

Abstract

Background: The ketogenic diet has been applied since the early 1900's for therapy. Although, the restrictive dietary application has proved to be effective in the treatment of neurologic disease we are seeing a more recent trend for the successful application of the ketogenic diet to treat metabolic disorders. The restrictive nature of the diet and the flu-like symptoms associated with its inception, however, make it intolerable for most patients.

Objectives: Here we report the mechanism by which the combination of an exogenous ketone, $\beta$-hydroxybutyrate (BHB) and butyric acid (BA) supplement (BHB-BA) can help facilitate ketosis with moderate and more tolerable dietary restriction by inducing ketogenesis and fatty acid oxidation.

Methods: HepG2 cell line was assayed for ketogenesis and fatty acid oxidation activity after treating the cells with equimolar dosing of BHB alone and BHB-BA in fed- and starved-conditions. HepG2, T98G, BV2 and HEK293 cells were analysed for Nrf2 expression through Western blotting after treatment with different BHB-BA concentrations.

Results: The results support incremental fatty acid oxidation and ketogenesis by BHB-BA over equimolar dosing of BHB on its own. We also show that the BHB-BA complex up-regulates expression of Nrf2, a master regulator of endogenous antioxidant proteins such as hemeoxygenase-1, glutathione peroxidase, superoxide dismutase- 1 and catalase, in a cell and dose specific manner.

Conclusion: Although more research needs to be done to explore the full metabolic and signalling activities by BHB and BA, the reported results provide an indication of how the short chain fatty acid, BA, could synergise the effects of the exogenous ketone body, BHB, and support the elevation of ketone status independent of or as an addition to the ketogenic diet.

Keywords: Ketogenic diet; Ketone supplements; Nutrition for neurological diseases; Ketogenesis; Ketosis; Low carb high fat diet; $\beta$-hydroxybutyrate (BHB); Butyric acid (BA)

\section{Introduction}

The ketogenic diet (KD), also known as low carb high fat (LCHF) diet is a low carbohydrate dietary program that includes an increased dietary fat intake designed to encourage the body to switch from using carbohydrate as a primary energy substrate to utilising fat [1]. The liver is capable of metabolizing mobilised fat to produce ketones $[2,3]$ that can be used by the mitochondria as an ATP substrate with what is said to be greater efficiency over glucose [4]. Although, the restrictive LCHF dietary application has proved effective in the treatment of neurologic disease [58] including non-tractable epilepsy $[9,10]$, we are seeing a more recent trend for the successful application of the ketogenic diet to treat metabolic disorders [11], including obesity [12,13], diabetes [14,15] and cardiovascular disease [16].

Nevertheless, the restrictive nature of the diet and the flulike symptoms associated with its inception make it intolerable for most patients. Supplementation with an exogenous ketone or ketone body salt, for instance, can support compliance of the restrictive dietary program and contribute to serum ketone load from a secondary source that adds to endogenous ketone production. This can improve tolerability of the restrictive diet and offset the energy drag associated with delayed endogenous ketone production and serum elevation $[17,18]$. This application 


\section{Current Research in Diabetes \& Obesity Journal}

was designed in light of the trending use of the ketogenic diet for the treatment of metabolic syndrome.

Butyric acid (BA), the short chain, fatty acid, may be able to play a strong supportive role as well. Research shows that supplementation with BA improves appetite control, insulin sensitivity and body mass proportions toward leaner measures $[19,20]$. One of the most interesting facts about BA pharmacology to fit right into the ketosis initiative is its induction of FGF21 in serum, liver and adipocytes to stimulate $\beta$-oxidation and endogenous ketone production [21]. $\beta$-oxidation of fats is a mechanism for ketone body production [22].

Here we report the metabolic influence on key features of ketone metabolism by a novel compound that combines the benefits of exogenous ketone body supplementation ( $\beta$-hydroxybutyrate, BHB) and $\mathrm{BA}$, the short chain fatty acid. The composition (BHB-BA) is designed with the intention of combining the known activities of the individual biochemicals and heightening the biological potential of each. This study was designed to validate the combination by assaying the effect of BHB-BA on ketone body production and fat metabolism compared with the ketone body (BHB) only, which is a common choice for exogenous ketone supplementation. The modulatory effect of the BHB-BA complex was also quantified in the context of, nuclear factor erythroid 2 (NFE2)-related factor 2 (Nrf2) activation. Nrf2 is a master regulator of antioxidant proteins like catalase, super oxide dismutase, glutathione peroxidase and hemeoxygenase-1. Our results show that the BHB-BA complex can induce a higher level of fatty acid oxidation and ketone body status (BHB) compared to BHB only. The complexed BHB-BA can also upregulate Nrf2 expression in a dose and cell specific manner.

\section{Methods}

\section{Cell culture}

BV2, HEK293, T98G and HepG2 cell lines were used in this study. BV2 and HEK293 cell lines were obtained from Dr. William Jia's (University of British Columbia) lab and T98G and HepG2 cells were obtained from Dr. Steven Pellech's (University of British Columbia) lab. BV2 and HEK293 cells were cultured in DMEM (Sigma, Oakville ON Canada) with 10\% FBS (Sigma) and $1 \%$ antimicrobial-antimycotic solution (Sigma). T98G and HepG2 cells were cultured in MEM (Sigma) with 10\% FBS (Sigma) and $1 \%$ antimicrobial-antimycotic solution. All the cells were cultured at $37{ }^{\circ} \mathrm{C}$ temperature incubator with $5 \% \mathrm{CO}_{2}$.

\section{Ketone synthesis assay}

HepG2 cells were plated on 96- well plate with a density of 10,000 cells per well and cultured with MEM media. When the cells were confluent the media was replaced with MEM media with no glucose and no serum. The MEM media was replaced from all wells of the 96 well plate of the HepG2 cells and add $100 \mu \mathrm{l}$ of the experimental media containing 3 different concentrations $(0.8,1.6$, and $2.4 \mathrm{mM})$ of BHB-BA and BHB in the
MEM media with and without serum. The level of ketones were measured in the media of the experimental wells at $0,30,60,90$ and 120 minutes time points with the digital Abbott's Freestyle monitor. For every data point $n=3$.

\section{Fatty acid oxidation assay}

HepG2 cells were plated on 96- well plate as described above with appropriate controls. When the cells were confluent the media was replaced with MEM media with low glucose and serum. The MEM media was replaced after $24 \mathrm{hrs}$ from the experimental wells of the 96 well plate of the HepG2 cells and add $100 \mu \mathrm{l}$ of the experimental media containing 3 different concentrations $(0.8,1.6,2.4 \mathrm{mM})$ of BHB-BA and BHB. A fatty acid oxidation assay kit from Luxcel Biosciences (Little Island, Cork, Ireland) has been used for this study. This kit supplies an external long chain fatty acid and uses flurometric method to determine depletion of oxygen from the surrounding media as a marker for fatty acid oxidation. After adding the substrate (long chain fatty acid) and fluorescent marker, all the wells were covered with mineral oil to prevent oxygen exchange from the environment. The data were recorded using Molecular Devices I3x at $0,15,30,45,60,75,90,105$ and 120 minutes. The data were calculated as "difference from 0 minute time point". For all data, $\mathrm{n}=3$.

\section{Treatment, protein isolation and western blot}

$0.032049 \mathrm{gm}$ of Branded BHBBA (BHB-BA, Rhema, Pitt Meadows, BC Canada) was added to $50 \mathrm{ml}$ of complete media (media varies according to the cell type) to make a final concentration of $4.0 \mathrm{mmol} / \mathrm{L} \mathrm{BHB}$ and $2 \mathrm{mmol} / \mathrm{L} \mathrm{BA}$. Cultured cells on tissue culture dish $\left(58 \mathrm{~cm}^{2}\right)$ until they are $90 \%$ confluent. On the day of the treatment, required volume of the premade treatment solutions of BHB-BA was added on to the existing media to make $0.8,1.6$, and $2.4 \mathrm{mmol} / \mathrm{L}$ final concentrations. After $30 \mathrm{mins}$ incubation the cells were washed with ice cold PBS. Then 100ul of lysis buffer (RIPA (Sigma) + protease inhibitor cocktail (100X, Sigma)) was added on each well. A scrapper was used to scrap the cells in to the lysis buffer and collect the samples in a $1.5 \mathrm{~mL}$ pre-chilled Eppendorf tube. Each sample was sonicated for 30 seconds followed by centrifugation for $13000 \mathrm{rpm}$ at $4^{\circ} \mathrm{C}$ for $30 \mathrm{mins}$. The supernatant was used for protein quantification (according to BioRad's DC Protein assay kit's instruction; BioRad Mississauga, ON Canada) followed by Western blotting. 15ug of protein was loaded for each of the samples for Western blot execution and 1:1000 concentration of primary Ab was used for Nrf2 (rabbit polyclonal anti-Nrf2 Ab; Abcam Inc Toronto, ON Canada) targeting. The membranes were scanned with the Quantity One scanner and the image were analysed with ImageJ software.

\section{Statistical analysis}

Data were analyzed through Student's T test for the ketogenesis assay and fatty acid oxidation assay. ANOVA was performed for all Nrf2 data analyses. For all cases $\mathrm{P}$ value smaller than 0.05 was considered as significant. 


\section{Current Research in Diabetes \& Obesity Journal}

Results

\section{BHB-BA can induce ketogenesis}
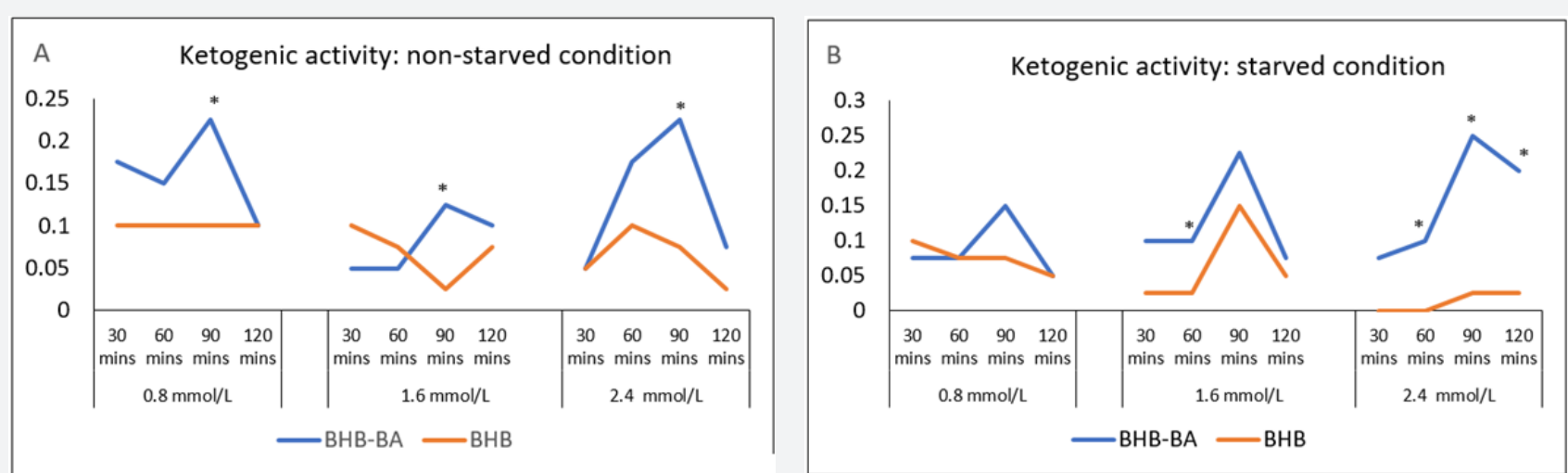

Figure 1: Ketogenesis in HepG2 cells after treating with three different concentrations of BHB-BA and BHB. Ketone production were measured from serum containing (A) and serum starved (B) media using a digital meter at 30,60, 90 and 120 minutes post incubation time points. Results are shown as the difference ketone production from the " 0 minute" time point to individual time points in different concentration. For all data, $n=3$. Data presented as mean and * represents statistical significance $(p<0.05)$.

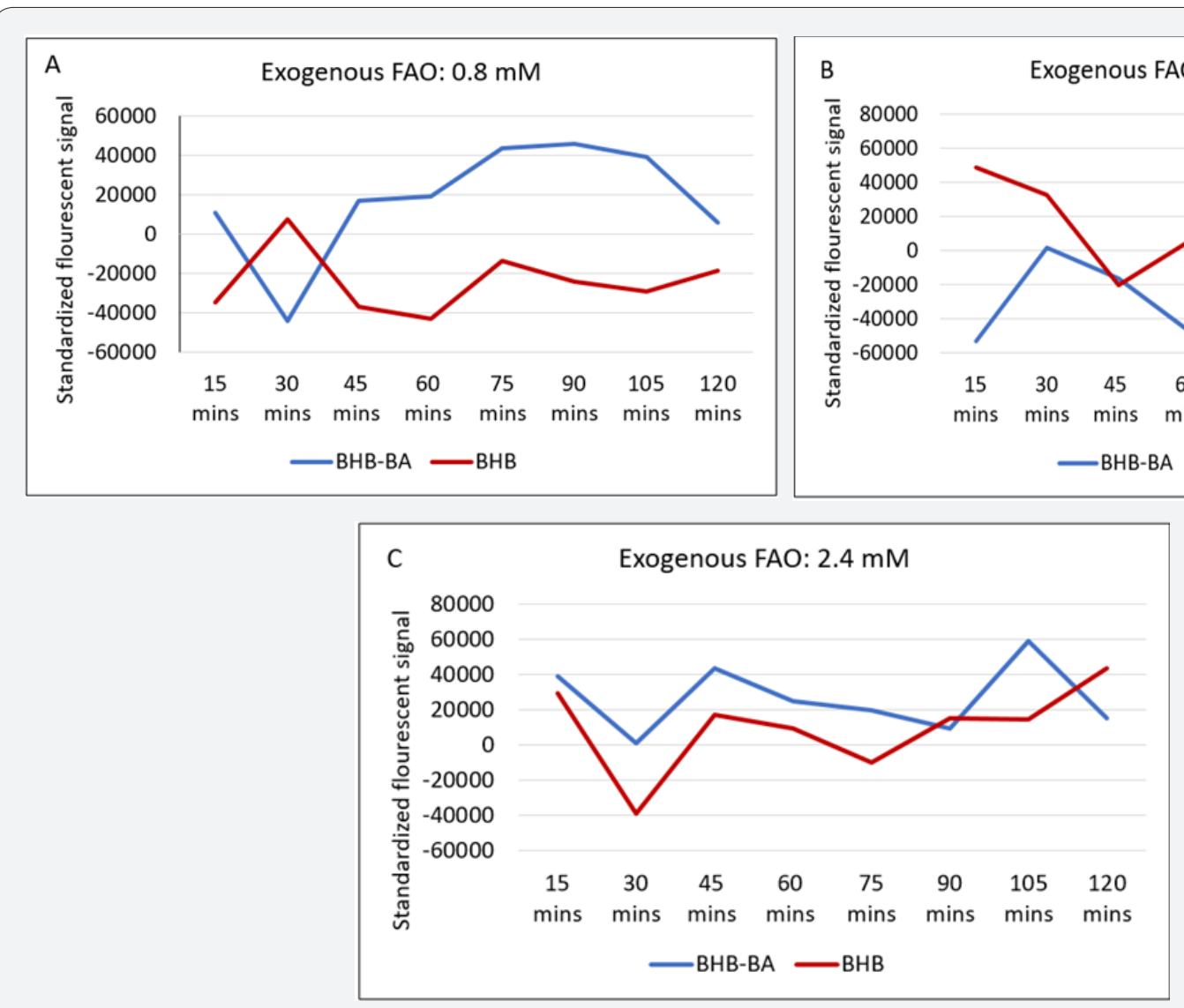

Figure 2: Fatty acid oxidation (FAO) activity in HepG2 cells after treating with three different concentrations $(0.8,1.6,2.4)$ of $\mathrm{BHB}$-BA and $\mathrm{BHB}$. FAO were measured through fluorometric assay (Luxcel Biosciences) using media containing exogenous long chain fatty acid and a fluorosence tagged oxygen quencher. Data were recorded at $0,15,30,45,60,75,90,105$, and 120 minutes and compared between BHB$\mathrm{BA}$ treated and BHB treated cells. Data were standardized to 0 minute and compared between BHB-BA and BHB for $0.8 \mathrm{mM}(\mathrm{A}), 1.6 \mathrm{mM}$ (B) and $2.4 \mathrm{mM}(\mathrm{C})$. For all data, $\mathrm{n}=3$.

Serum and glucose starved hepatic cell line (HepG2) was used in this study to investigate whether the combination of BHB and $\mathrm{BA}$ can induce ketogenesis under starving conditions. BHB alone was also tested to compare against BHB-BA activity. The synergy between BHB and BA appears to result in better ketogenic activity in both serum non-starved (Figure 1A) and starved (Figure 1B) conditions compare to equimolar BHB concentrations alone. In both cases the highest degree of ketogenic activity was observed at the 90 minute time point across the variable concentrations. While the difference was significant for all $(\mathrm{p}=0.04,0.03$, and 
0.005 for $0.8,1.6$ and $2.4 \mathrm{mmol} / \mathrm{L}$ ) concentrations, respectively in the non-starved condition, only significantly $(\mathrm{p}=0.00692)$ higher ketogenesis was observed at the $2.4 \mathrm{mmol} / \mathrm{L}$ concentration in the starved condition. During starvation, the ketone bodygenerating activity was recorded to be slightly higher than the non-starved condition at the higher concentration of BHB-BA (1.6 and $2.4 \mathrm{mM}$ ). This outcome indicates a plausible additive ketogenic activity induced by starvation. The concentration differences of BHB-BA does not seem to play a role in the production of ketones in the non-starved state. However, in the starved condition a higher concentration does seem to have an incremental effect on ketone generation by BHB-BA but this is not the case in the BHB-only group (Figure 1B).

\section{BHB-BA can modulate lipid metabolism by hepatic cells}

Since we observed higher ketone levels in the media after adding BHB-BA, we wanted to investigate the source of the elevated ketone. Serum and glucose starved hepatic cell line (HepG2) was used to investigate whether BHB-BA can induce lipid metabolism and fatty acid oxidation (FAO) and how this rate compares to equimolar levels of BHB on its own (Figure 2). Endogenous fatty acid oxidation is a pivotal step in weight management program that effectively diminishes stored fat. It is also the mechanism by which ketone bodies are generated from fat as metabolic intermediates that can subsequently be used as energy substrates for ATP generation by cells like neurons. At 0.8 (Figure 2A,B) and $2.4 \mathrm{mM}$ (Figure $2 \mathrm{C}$ ) BHB concentration, the BHB-BA combinations show higher FAO capacity compared to treatment with equimolar BHB alone at almost all the time points (non-significant). More importantly BHB-BA shows higher FAO than the control ( 0 minute) at most time points, whereas BHB treatment could not produce enough FAO to surplus 0-minute time point in most cases. At $1.6 \mathrm{mM}$ BHB concentration, at most of the time points, both BHB and BHB-BA failed to perform higher FAO compare to the starting point ( 0 minute). However, at the 105- and 120-minute time points, BHB-BA has a higher FAO activity than BHB only treatment. At 15- and 90-minutes time point the pattern was reversed (non-significant).

\section{Modulation of cellular antioxidative pathways}
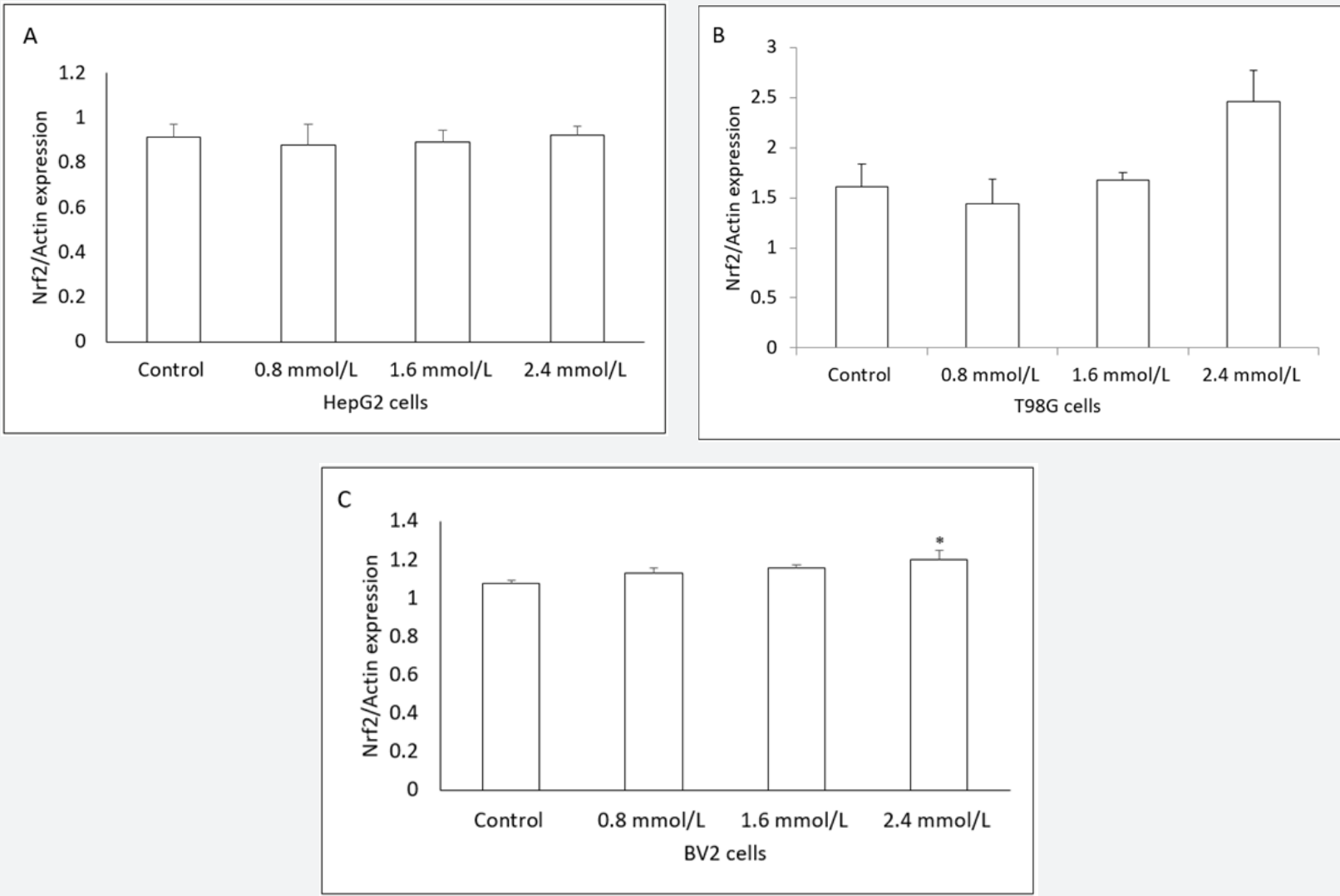

Figure 3: Western Blot results of NRf2 expression standardized to corresponding samples' Actin expression in HepG2 (A), T98G (B), BV2 (C), and HEK293 (D) cells after treated with 3 different concentrations of BHB-BA. For each samples, $n=3$. Data presented as mean + SEM (Standard error of the mean) and * represents statistical significance $(p<0.05)$.

In addition to BHB-BA's ketogenesis and fatty acid oxidation activities, the role of BHB-BA in other cellular pathways that may be relevant to cell health was also investigated. Oxidative damage could put a toll on overall health during strenuous weight loss programs and athletic performance. The effect of BHB-BA on cellular antioxidative pathways through Nrf2 modulation was investigated on a number of cell lines and the HepG2 cell line was the first pick since this cell line showed improved 


\section{Current Research in Diabetes \& Obesity Journal}

metabolic activity in our previous experiments. Three other cell lines, BV2 microglia, a representative of immune system; T98G glioblastoma, representative of nervous system and HEK293 cells, a representative of peripheral tissue, were also studied. As this study was performed to assess the therapeutic potential of the formula (BHB-BA), no comparative analysis was done with BHB alone. HepG2 cells did not demonstrate any significant changes in Nrf2 expression in response to varying BHB-BA concentration (Figure 3A).

However, a concentration dependent expression pattern was observed in BV2, HEK293 and T98G cells (Figure 3B-D), where Nrf2 expression was elevated in a concentration-dependent manner. At $2.4 \mathrm{mmol} / \mathrm{L}$ concentration, both T98G cells $(\mathrm{p}=0.06)$ and BV2 ( $\mathrm{p}=0.0132)$ cells display higher Nrf2 expression compared to control cells.

\section{Discussion}

The known signalling activities by BA played a significant motivational role in our design of experimentation that could suggest the synergistic application of BHB with BA in the context of fatty acid oxidation, ketone synthesis and the facilitation of ketosis. It is well established that exogenous ketone supplementation can play a role in ketosis and therapy $[23,24]$. However, it is important to explore how endogenously activated ketogenesis could be induced by signalling activity independent of dietary changes to support the metabolic activity needed to help activate and maintain endogenously generated ketosis. Our experimental results show that administration of BA with BHB results in incremental fatty acid oxidation over equimolar BHB administration alone. Furthermore, we also show an increased BHB status in media for the BA accompanied BHB treatment versus equimolar $\mathrm{BHB}$ alone. This supports our application of BHB-BA as a more effective mechanism over BHB alone to induce fatty acid oxidation and ketosis; and, furthermore, maintain ketogenesis to support endogenous synthesis of BHB by oxidation of fatty acids from endogenous lipids.

The objective of the reported strategy and experimental results here is to help define a mechanism by which the benefits of the ketogenic diet might be enhanced or facilitated by an exogenous BHB source that helps facilitate adherence to a healthy dietary program but does not negatively inhibit lipolysis or oxidation of endogenous fat. True ketogenesis can be averted by the administration of high dose oral BHB as a function of lipolysis inhibition [25], while BA helps maintain fatty acid oxidation signalling to support ketogenesis [26,27]. This BHB-BA signalling, and the fact that BA also improves insulin sensitivity [28] to support serum glucose clearance is conducive to supporting nutritional ketosis by ketogenesis and may emulate, better than BHB alone, the mechanism by which the ketogenic diet works systemically. Furthermore, the stimulatory influence of the BHB-BA complex on Nrf2 presents, yet, another layer of tremendous value in terms of cell protection that will need to be explored more thoroughly.
Nrf2 induction is shown to increase cellular defense protecting neurons during toxicity and associated mitochondrial stress to the point of reduced lesion development $[29,30]$. Nrf2 induction protects cells from inflammatory activity and mortality [31,32]. Nrf2 signaling pathways are showing promise as a counteraction to mitochondrial dysfunction in Parkinson's disease [32]. Nrf2 induction is shown to convey critical defense against elevated serum-glucose induced oxidative injury to cardiac muscle cells [33]. One of the major limitations of this study was the execution of the experiments on different cell lines where some of the lines are, in fact, cancer lines. However, experiments were conducted on different well-studied in vitro models that represent different physiological systems in our body. BHB-BA will need to be utilised for clinical study in large populations in order for us to know the full performance and medicinal therapeutic and preventive potential of this novel strategy. Nevertheless, these studies are pointing to the fact that we might be on the right track with BHB-BA strategy as a more effective treatment strategy over BHB alone for various health conditions including weight mismanagement where the endogenous induction of fatty acid oxidation and ketogenesis can play a corrective role.

\section{References}

1. Cavaleri F, Bashar E (2018) Potential Synergies of $\beta$-Hydroxybutyrate and Butyrate on the Modulation of Metabolism, Inflammation, Cognition, and General Health. Journal of Nutrition and Metabolism 2018: 13 .

2. Neat CE, Thomassen MS, Osmundsen $H$ (1980) Induction of peroxisomal beta-oxidation in rat liver by high-fat diets. Biochemical Journal 186(1): 369-371.

3. Kaikaus RM, Chan W, Lysenko N, Ray R, de Montellano PO, et al. (1993) Induction of peroxisomal fatty acid beta-oxidation and liver fatty acid-binding protein by peroxisome proliferators. Mediation via the cytochrome P-450IVA1 omega-hydroxylase pathway. Journal of Biological Chemistry 268(13): 9593-9603.

4. Yudkoff M, Daikhin Y, Melø TM, Nissim I, Sonnewald U, et al. (2007) The ketogenic diet and brain metabolism of amino acids: relationship to the anticonvulsant effect. Annu Rev Nutr 27: 415-430.

5. Livingston S, Pauli LL (1975) Ketogenic diet and epilepsy. Developmental Medicine \& Child Neurology 17(6): 818-819.

6. Neal EG, Chaffe H, Schwartz RH, Lawson MS, Edwards N, et al. (2008) The ketogenic diet for the treatment of childhood epilepsy: a randomised controlled trial. The Lancet Neurology 7(6): 500-506.

7. Rho JM (2008) The ketogenic diet and epilepsy. Current Opinion in Clinical Nutrition \& Metabolic Care 11(2): 113-120.

8. Masino S, Kawamura M, Wasser C, Pomeroy L, Ruskin D (2009) Adenosine, ketogenic diet and epilepsy: the emerging therapeutic relationship between metabolism and brain activity. Current neuropharmacology 7(3): 257-268.

9. Ismayilova N, Leung MA, Kumar R, Smith M, Williams RE (2018) Ketogenic diet therapy in infants less than two years of age for medically refractory epilepsy. Seizure 57: 5-7.

10. Kossoff EH, Zupec-Kania BA, Rho JM (2009) Ketogenic diets: an update for child neurologists. J Child Neurol 24(8): 979-988.

11. Sharman MJ, Kraemer WJ, Love DM, Avery NG, Gómez AL, et al. (2002) A ketogenic diet favorably affects serum biomarkers for cardiovascular 


\section{Current Research in Diabetes \& Obesity Journal}

disease in normal-weight men. The Journal of nutrition 132(7): 18791885.

12. Foster GD, Wyatt HR, Hill JO, McGuckin BG, Brill C, et al. (2003) A randomized trial of a low-carbohydrate diet for obesity. New England Journal of Medicine 348(21): 2082-2090.

13. Johnstone AM, Horgan GW, Murison SD, Bremner DM, Lobley GE (2008) Effects of a high-protein ketogenic diet on hunger, appetite, and weight loss in obese men feeding ad libitum. The American journal of clinical nutrition 87(1): 44-55.

14. Westman EC, Yancy WS, Mavropoulos JC, Marquart M, McDuffie JR (2008) The effect of a low-carbohydrate, ketogenic diet versus a lowglycemic index diet on glycemic control in type 2 diabetes mellitus. Nutrition \& metabolism 19(5): 36.

15. Yancy WS, Foy M, Chalecki AM, Vernon MC, Westman EC (2005) A low-carbohydrate, ketogenic diet to treat type 2 diabetes. Nutrition \& metabolism 2(1): 34

16. Bueno NB, de Melo ISV, de Oliveira SL, da Rocha Ataide T (2013) Verylow-carbohydrate ketogenic diet $\mathrm{v}$. low-fat diet for long-term weight loss: a meta-analysis of randomised controlled trials. British Journal of Nutrition 110(7): 1178-1187.

17. Plecko B, Stoeckler-Ipsiroglu S, Schober E, Harrer G, Mlynarik V, et al. (2002) Oral beta-Hydroxybutyrate Supplementation in Two Patients with Hyperinsulinemic Hypoglycemia: Monitoring of betaHydroxybutyrate Levels in Blood and Cerebrospinal Fluid, and in the Brain by In Vivo Magnetic Resonance Spectroscopy. Pediatric research 52(2): 301-306.

18. White H, Venkatesh B (2011) Clinical review: ketones and brain injury Critical Care 15(2): 219.

19. Canfora EE, Jocken JW, Blaak EE (2015) Short-chain fatty acids in control of body weight and insulin sensitivity. Nature Reviews Endocrinology 11(10): 577-591.

20. Darzi J, Frost GS, Robertson MD (2011) Do SCFA have a role in appetite regulation? Proceedings of the Nutrition Society 70(1): 119-128.

21. Li H, Gao Z, Zhang J, Ye X, Xu A, et al. (2016) Sodium butyrate stimulates expression of fibroblast growth factor 21 in liver by inhibition of histone deacetylase 3. Diabetes 61(4): 797-806.

22. McGarry J, Foster D (1980) Regulation of hepatic fatty acid oxidation and ketone body production. Annual review of biochemistry 49(1): 395-420.

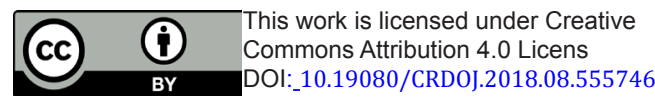

23. D Agostino DP, Pilla R, Held HE, Landon CS, Puchowicz M, et al. (2013) Therapeutic ketosis with ketone ester delays central nervous system oxygen toxicity seizures in rats. American Journal of PhysiologyRegulatory, Integrative and Comparative Physiology 304(10): R829-R36.

24. Kesl SL, Poff AM, Ward NP, Fiorelli TN, Ari C, et al. (2016) Effects of exogenous ketone supplementation on blood ketone, glucose, triglyceride, and lipoprotein levels in Sprague-Dawley rats. Nutrition \& metabolism 13(1): 9.

25. Laffel L (1999) Ketone bodies: a review of physiology, pathophysiology and application of monitoring to diabetes. Diabetes/metabolism research and reviews 15(6): 412-426.

26. Zhang X, Yeung DC, Karpisek M, Stejskal D, Zhou ZG, et al. (2008) Serum FGF21 levels are increased in obesity and are independently associated with the metabolic syndrome in humans. Diabetes 57(5): 1246-1253.

27. Hird F, Symons R (1962) The mechanism of ketone-body formation from butyrate in rat liver. Biochemical Journal 84(1): 212-216.

28. Gao Z, Yin J, Zhang J, Ward RE, Martin RJ, et al. (2009) Butyrate improves insulin sensitivity and increases energy expenditure in mice. Diabetes 58(7): 1509-1517.

29. Shih AY, Imbeault S, Barakauskas V, Erb H, Jiang L, et al. (2005) Induction of the Nrf2-driven antioxidant response confers neuroprotection during mitochondrial stress in vivo. Journal of Biological Chemistry 280(24): 22925-22936.

30. Lee JM, Shih AY, Murphy TH, Johnson JA (2003) NF-E2-related factor-2 mediates neuroprotection against mitochondrial complex I inhibitors and increased concentrations of intracellular calcium in primary cortical neurons. Journal of Biological Chemistry 278(39): 3794837956.

31. Thimmulappa RK, Scollick C, Traore K, Yates M, Trush MA, et al. (2006) Nrf2-dependent protection from LPS induced inflammatory response and mortality by CDDO-Imidazolide. Biochemical and biophysical research communications 351(4): 883-889.

32. Tufekci KU, Civi Bayin E, Genc S, Genc K (2011) The Nrf2/ARE pathway: a promising target to counteract mitochondrial dysfunction in Parkinson's disease. Parkinson's disease 2011: 314082.

33. He X, Kan H, Cai L, Ma Q (2009) Nrf2 is critical in defense against high glucose-induced oxidative damage in cardiomyocytes. Journal of molecular and cellular cardiology 46(1): 47-58.

\section{Your next submission with Juniper Publishers will reach you the below assets}

- Quality Editorial service

- Swift Peer Review

- Reprints availability

- E-prints Service

- Manuscript Podcast for convenient understanding

- Global attainment for your research

- Manuscript accessibility in different formats

( Pdf, E-pub, Full Text, Audio)

- Unceasing customer service

Track the below URL for one-step submission

https://juniperpublishers.com/online-submission.php 